\section{SOI: 1.1 /TAS DOI: $10.15863 /$ TAS International Scientific Journal Theoretical \& Applied Science}

p-ISSN: 2308-4944 (print) e-ISSN: 2409-0085 (online)

Year: $2016 \quad$ Issue: 2 Volume: 34

Published: 29.02.2016 $\underline{\text { http://T-Science.org }}$

SECTION 7. Mechanics and machine construction.
Rana Gh. Faysal Master student, Engineer, The ministry of communications, Iraq

Eng.rana3000@yahoo.com
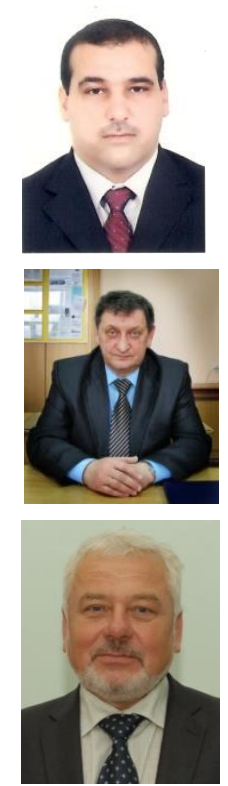

Auday Shaker Hadi Assistant Lecturer, Ph.D student, University of Technology,

Iraq aodayshaker@mail.ru

Aleksandr Nikolaevich Litvinov Doctor of Technical Sciences, Professor, Penza State University, Russia

aleksletvinov@mail.ru

Valery Yakovlevich Bannov Doctor of Technical Sciences, Professor, Penza State University, Russia bvy@bk.ru

\title{
ANALYSIS OF THERMAL FIELDS OF PRINTED CIRCUIT ASSEMBLIES
}

\begin{abstract}
Modeling of the stress-strain state (SSS) of the basic structural elements of radio electronics (RE) in the conditions actual operation is an urgent problem, whose solution allows the early stages of design to identify the most loaded elements of radio electronics (RE) and receive Design Technological solutions designed to optimize the design. The aim of this work is to increase the reliability and providing the required performance characteristics of product design RES technological method. A mathematical model of a real board RE with hinged elements that produce heat and under the influence of the operating mode of the heat. Mathematical modeling of the thermal field and SSS board based on the finite element method (FEM), implemented in the software package ANSYS. Achieved Mathematical modeling and analysis of thermal fields and SSS boards under the action of external thermal performance impacts. Most loaded area set board in which defects may develop mechanical origin, including latent conditions in RE. Proposed design -technological methods to reduce stress and improve thermo mechanical reliability design. The proposed model allows us to investigate the SSS of the boards with the real location and conditions of the outboard elements on it. We establish by mathematical modeling of the most loaded zone board and its components under operational thermal effects.

Key words: printed assembly, printed board, radio electronics, thermal effect, the temperature field, the stressstrain state.

Language: Russian

Citation: Faysal RG, Hadi AS, Litvinov AN, Bannov VY (2016) ANALYSIS OF THERMAL FIELDS OF PRINTED CIRCUIT ASSEMBLIES. ISJ Theoretical \& Applied Science, 02 (34): 58-65.

Soi: http://s-o-i.org/1.1/TAS-02-34-10 Doi: crossef http://dx.doi.org/10.15863/TAS.2016.02.34.10
\end{abstract}

\section{АНАЛИЗ ТЕПЛОВЫХ ПОЛЕЙ ПЕЧАТНЫХ УЗЛОВ}

Аннотация: Моделирование напряженно-деформированного состояния (НДС) основных элементов конструкций радиоэлектроники (РЭ) в условиях реальной эксплуатациии является актуальной проблемой, решение которой позволяет на ранних этапах проектирования выявлять наиболее нагруженные элементы РЭ и принимать конструкторско- технологические решения для оптимизации проектируемых конструкции. Целью работы является повышение надежности и обеспечение требуемых тактикотехнических характеристик изделий РЭ конструкторско- технологическими способими. Рассмотрена математическая модель теплового поля реального печатного узла ПУ РЭ который состоит из печатной платы с навесными элементами, излучающими тепло и находящейся под действием эксплуатационного 
теплового режима. Математическое моделирование теплового поля и НДС печатной платы (НДС ПП) основано на применении метода конечных элементов (МКЭ), реализованного в программном комплексе ANSYS. Выполнено математическое моделирование и анализ тепловых полей и НДС ПП при действии эксплуатационных внешних тепловых воздействий. Установлены наиболее нагруженные зоны ПП, в которых могут развиваться дефекты механического происхождения, в том числе и латентные, в условиях эксплуатации РЭ. Предложены конструкторско -технологические методы по снижению термомеханических напряжений $u$ повышению надежности конструкции. Предложенная модель позволяет исследовать НДС ПП с учетом реального расположения и условий установии навесных элементов на ней. Путем математического моделирования устанавлены наиболее нагруженные зоны ПП и ее элементов при эксплуатациионих тепловых воздействиях.

Ключевые слова: печатной узел, печатной плата, радиоэлектроника, тепловое воздействие, поле температур, напряженно-деформированное состояние.

Современный уровень развития электронных технологий характеризуется тем, что, с одной стороны, растут мощности и увеличивается плотность размещения элементов, a c другой - снижается стойкость электрорадиоэлементов к тепловым и механическим воздействиям. Все сложнее обеспечить требуемые температурный и вибрационный режимы для стабильной работы РЭ на основе традиционных методов.

Одной из важнейших задач развития научных основ конструирования и технологии изготовления блоков и изделий современных РЭ и различных приборных устройств является создание адекватных прикладных моделей, описывающих состояние конструкций и отдельных элементов в процессе их производства, испытаний и эксплуатации [1].

Системный анализ различных конструкций РЭ и приборных устройств показывает, что большинство изделий, блоков и их элементов являются гетерогенными структурами, подверженными внешним воздействиям. За счет изменения их напряженно-деформированного состояния (НДС) происходит изменение основных электропараметров устройств, ухудшение метрологических характеристик, снижение их надежности, а в ряде случаев их частичный или полный отказ в результате разрушения отдельных элементов. При этом основными являются динамические и температурные воздействия, возникающие в ходе выполнения различных технологических операций в производстве, при испытаниях и в процессе эксплуатации изделий в широком диапазоне тепловых и вибрационных нагрузок [1, $2]$.

В процессе технологического проектирования требуется полный анализ технологических факторов, определяющих качество конечного продукта. Отметим, что основным источником отказов РЭ являются печатные платы, которые служат основой в большинстве современных электронных систем. Технологические аспекты повышения качества печатных плат находятся на острие современных теоретических и научно - практических исследований.

Анализ технологических процессов показывает, что существующие модели [3] не в полной мере учитывают явления и процессы, происходящие в гетерогенных структурах изделий и нуждаются в уточнении и совершенствовании [4].

\section{1. Модель для исследования}

Существующие модели для исследования полей термомеханических напряжений в гетерогенной структуре платы, как правило, не учитывают ряда конструктивных особенностей исследуемой платы: наличие электро радиоэлементов (ЭРЭ) на её поверхности, способы их крепления к плате, наличие дополнительних клеевых или паяных швов между основаними ЭРЭ и поверхностью платы, разогрев отдельных участков платы за счет мощностей, выделяемых некоторыми ЭРЭ и т. п. [3].

Существующие аналитические методы расчета часто приводят к достаточно грубым оценкам тепловых полей и термомеханических напряжений, возникающих в платах, а в ряде случаев могут приводить к ошибочным результатам и выводам.

Рассмотрим печатный узел блока РЭ, представленный на рис.1. На поверхности прямоугольной платы с размерами $(a \times b)$ в плане и толщиной $h$ расположены ЭРЭ, имеющие различные размеры и массы. ПУ крепится к корпусу блока в пяти точках винтами (по углам и вблизи центра платы).

В таблице 1 приведены мощности, выделяемые активными ЭРЭ 


\begin{tabular}{|c|c|c|c|c|c|c|}
\hline Impact Factor: & $\begin{array}{l}\text { ISRA (India) } \\
\text { ISI (Dubai, UAF } \\
\text { GIF (Australia) } \\
\text { JIF }\end{array}$ & $\begin{array}{l}=1.344 \\
=0.829 \\
=0.564 \\
=1.500\end{array}$ & $\begin{array}{l}\text { SIS (USA) } \\
\text { PИНЦ (Russia) } \\
\text { ESJI (KZ) } \\
\text { SJIF (Morocco) }\end{array}$ & $\begin{array}{l}=0.912 \\
=0.179 \\
=1.042 \\
=2.031\end{array}$ & $\begin{array}{l}\text { ICV (Poland) } \\
\text { PIF (India) }\end{array}$ & $\begin{array}{l}=6.630 \\
=1.940\end{array}$ \\
\hline
\end{tabular}

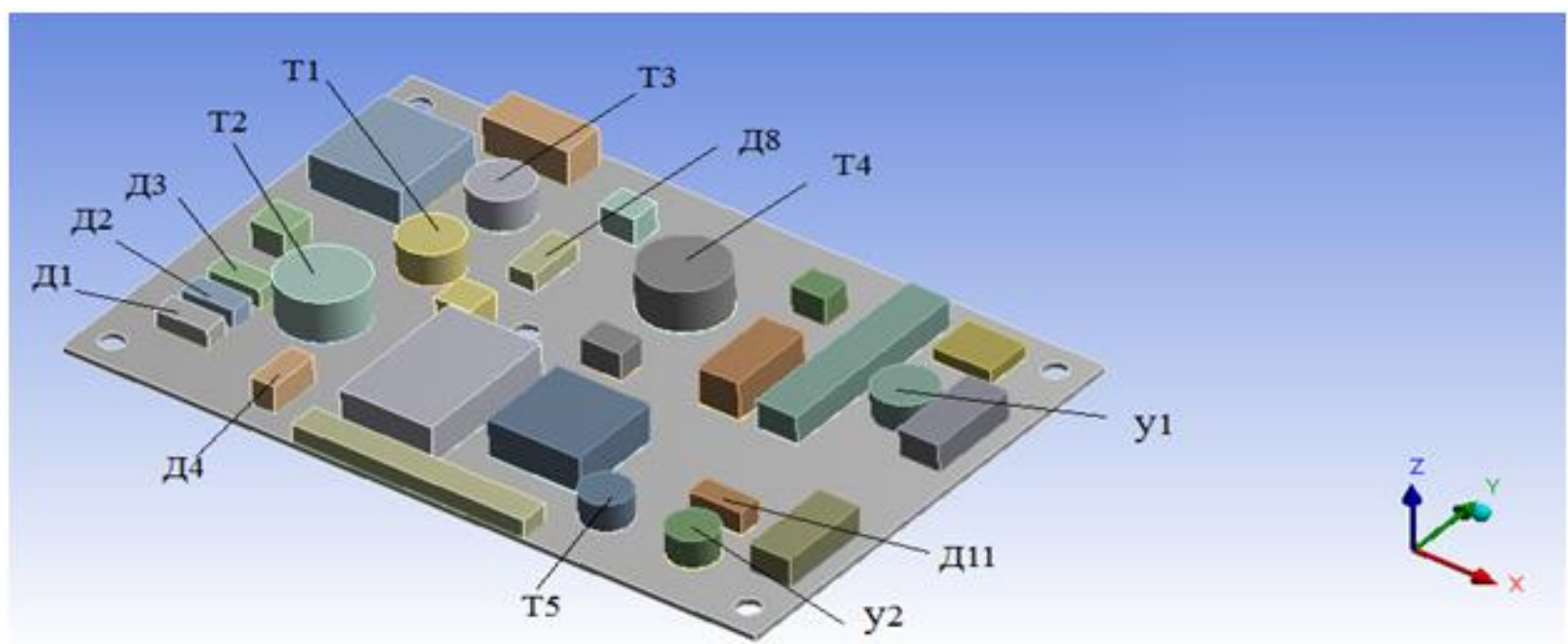

Рисунок 1- Печатный узел.

Таблица 1

Мошности, выделяемые активными ЭРЭ.

\begin{tabular}{|c|c|c|c|c|c|c|c|}
\hline $\begin{array}{c}\text { Обозначение активного } \\
\text { элемента }\end{array}$ & $\mathrm{T}_{1}$ & $\mathrm{~T}_{2}, \mathrm{~T}_{4}$ & $\mathrm{~T}_{3}, \mathrm{~T}_{5}$ & $\mathrm{y}_{1}, \mathrm{y}_{2}$ & $Д_{1}-Д_{4}$ & $Д_{8}$ & $Д_{11}$ \\
\hline Выделяемая мощность, Вт & 0,2 & 1,1 & 0,1 & 0,08 & 0,06 & 0,077 & 0,052 \\
\hline
\end{tabular}

При анализе теплового поля ПУ были рассмотрены моделей:

I - ЭРЭ жестко закреплены на плате, т.е. припаяны (приклеены) поверхности ПП;

II - элементы представляются в виде точечных источников тепла.

Задача по определению поля температур на ПП решается методом конечных элементов с использованием процедуры автоматического разбиения платы на конечные элементы. Так как плата является статически неопределимой системой, то любое изменение температуры на её поверхности приводит к ее деформированию, которое сопровождается изгибом платы. Напряженно-деформированное состояние платы характеризуется интенсивностью деформаций $\varepsilon_{i}$, прогибами $W$ и эквивалентными напряжениями $\sigma_{э к в}$, которые можно определить в соответствии с энергетическим критерием Мизеса $[7,8]$ :

$$
\begin{gathered}
\sigma_{\text {экв }}=\sqrt{\frac{1}{2}\left[\left(\sigma_{1}-\sigma_{2}\right)+\left(\sigma_{1}-\sigma_{3}\right)+\left(\sigma_{2}-\sigma_{3}\right)\right]},(1) \\
\text { где } \sigma_{1}, \sigma_{2} \cdot \sigma_{3}-\text { главные напряжения. }
\end{gathered}
$$

В табл. 2 представлены численные результаты математического моделирования теплового поля (расчетные значения температур) на поверхности платы для двух моделей. При расчете приняты следующие исходные данные: материал платы стеклотекстолит; $(a \times b)=(120 \times 80) . \mathrm{mм}^{2}, \quad h=1.5 \quad$ мм; температура окружающей среды $\mathrm{T}_{0}=22{ }^{\circ} \mathrm{C}$. В первой модели считается, что элементы припаяны к ПП припоем ПОС61 с толщиной шва $h_{u}=0,1 \mathrm{мм}$.

ПП находится в рабочем режиме в соответствии с данными табл.1, температуры указаны в точках расположения активных элементов.

\section{Значения температур на поверхности ПП.}

\begin{tabular}{|c|c|c|c|c|c|c|c|c|c|c|c|}
\hline \multirow[t]{2}{*}{ Модель } & \multicolumn{11}{|c|}{ Активный элемент, температура $\mathrm{T},{ }^{\circ} \mathrm{C}$} \\
\hline & $\mathrm{T}_{1}$ & $\mathrm{~T}_{2}$ & $\mathrm{~T}_{3}$ & $\mathrm{~T}_{4}$ & $\mathrm{~T}_{5}$ & $\mathrm{Y}_{1}$ & $\mathrm{y}_{2}$ & $\begin{array}{l}Д_{1}, \\
Д_{2}, \\
\text { Д }_{3}\end{array}$ & $Д_{4}$ & Д8 & $Д_{11}$ \\
\hline $\mathrm{I}$ & 23,8 & 28,4 & 22,9 & 28,4 & 23,2 & 22,6 & 23,2 & 22,7 & 23,0 & 22,7 & 22,7 \\
\hline II & 24,2 & 28,4 & 23,1 & 27,6 & 22,9 & 22,0 & 22,8 & 22,6 & 23,2 & 22,7 & 22,7 \\
\hline
\end{tabular}

Таблица 2 
Анализ результатов расчета по первой и второй моделям показал, что выбор модели несущественно влияет на распределение температур по поверхности ПП в рабочем режиме активных элементов. В тоже время математическое моделирование НДС ПП показало, что при полученном распределении температур полный анализ НДС возможен только при использовании первой модели, которая учитывает существенное изменение жесткости платы за счет ее связи с элементами. При этом на поверхности платы термомеханические напряжения не превышают 0,3 МПа. Наиболее нагруженными оказываются паяные швы под активным элементом $\mathrm{T}_{2}$, где $\sigma_{\text {экв }}=0.9 \mathrm{MПа}$.

\section{2. Моделирование состояние ПП при} тепловых воздействиях

Рассмотрим состояние ПП, представленной на рис.1, при её эксплуатации. В соответственно с ТУ она эксплуатируется в диапазоне температур от $-50{ }^{\circ} \mathrm{C}$ до $+85^{\circ} \mathrm{C}$. По прежнему рассматриваем две модели. Моделирование НДС ПП выполнено метода конечных элементов, реализованном в пакете прикладных программ ANSYS.

На рис. 2 и 3 представлено НДС платы с элементами при действии температуры $\mathrm{T}=85^{\circ} \mathrm{C}$ и $\mathrm{T}=-50{ }^{\circ} \mathrm{C}$, соответсвенно. Плата находится в рабочем состоянии, т.е. активные элементы выделяют мощность в соответствии с данными

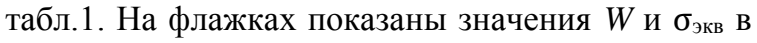
некоторых точках платы.

Из анализа полученных результатов следует, что при тепловом эксплуатационном воздействии происходит деформация платы, сопровождающияся её изгибом. При этом для рассматриваемой конструкции максимальные прогибы составляют: $\max W=0,0201$ мм при $\mathrm{T}=85^{\circ} \mathrm{C}$ и $\max W=0,0218$ мм при действии температуры $\mathrm{T}=-50^{\circ} \mathrm{C}$ (рис.2а и $\left.3 \mathrm{a}\right)$.

Анализ поля эквивалентных напряжений $\sigma_{\text {экв }}$ (рис.2б и3б) показывает, что наиболее нагруженные зоны при тепловом воздействии расположены в зоне элемента $\mathrm{T}_{4}$ (см.рис.1) и в зонах угловых отверстий, выполненных в плате для её крепления к блоку.

Максимальные напряжения в зоне элемента $\mathrm{T}_{4}$ возникают в паяном шве и составляют $\max$ $\sigma_{\text {экв }}=103,51 \mathrm{MПа} \mathrm{при} \mathrm{T}=85^{\circ} \mathrm{C}$ и $\max \sigma_{\text {экв }}=116,69$ МПа при $\mathrm{T}=-50^{\circ} \mathrm{C}$. В зоне угловых отверстий $\max \sigma_{\text {экв }}=49,38$ МПа и 55,28 МПа при $\mathrm{T}=85^{\circ} \mathrm{Cи}$ $\mathrm{T}=-50^{\circ} \mathrm{C}$, соответственно.

Для припоя ПОС 61 предел прочности составляет [5] $\sigma_{\text {в }}=47$ МПа, а для материала платы $\sigma_{\mathrm{B}}=350$ МПа. Наличие больших напряжений в указанных зонах может приводить к возникновеню пластических деформаций в паяных швах и образованию микротрещин в зоне отверстий под крепежные винты, а также к развитию латентных дефектов в виде скрытых непропаев, микротрещин и иных дефектов, которые могут развиваться в наиболее нагруженных зонах при дополнительном вибрационном воздействии в процессе эксплуатации.

На рис .4 и 5 предоставлены НДС платы при тепловом воздействии в соответствии с второй моделью, которая не учитывает изменение жесткости платы за счет ЭРЭ, расположенных на ее поверхности.

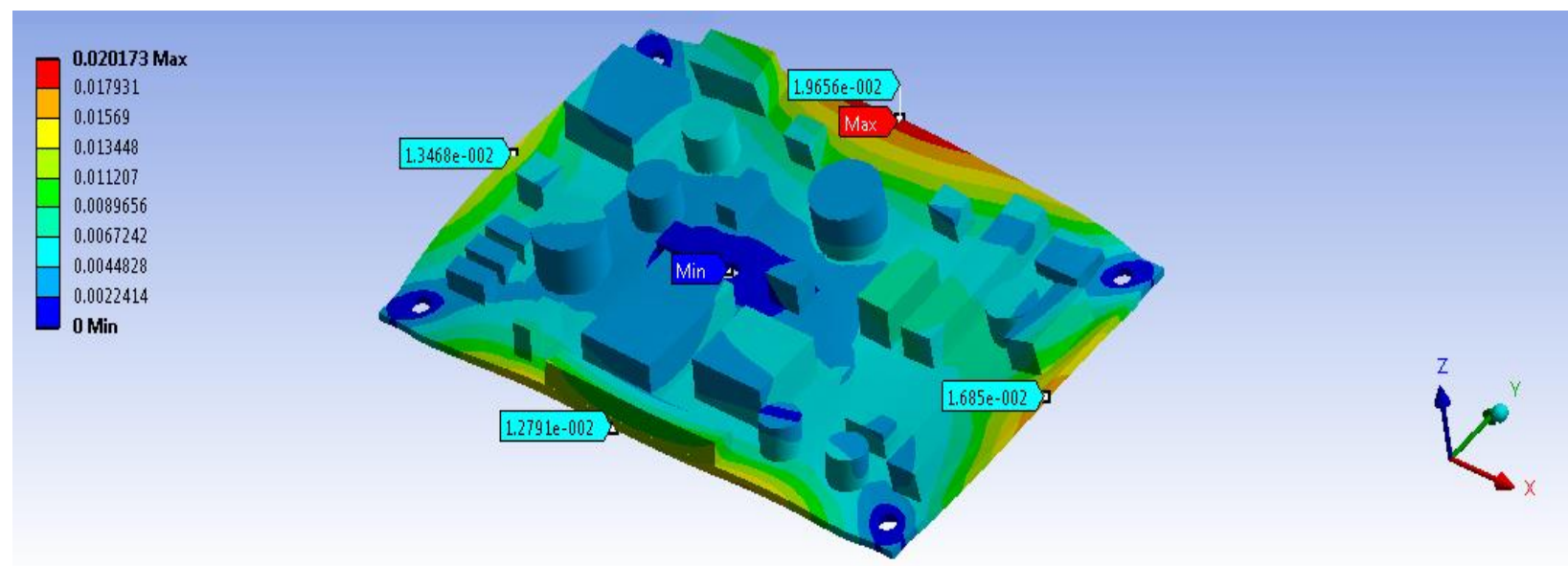




\begin{tabular}{l|lr|ll|ll} 
& ISRA (India) & $=\mathbf{1 . 3 4 4}$ & SIS (USA) & $=\mathbf{0 . 9 1 2}$ & ICV (Poland) & $=\mathbf{6 . 6 3 0}$ \\
Impact Factor: & ISI (Dubai, UAE) $=\mathbf{0 . 8 2 9}$ & PUHL (Russia) $=\mathbf{0 . 1 7 9}$ & PIF (India) & $=\mathbf{1 . 9 4 0}$ \\
& GIF (Australia) & $=\mathbf{0 . 5 6 4}$ & ESJI (KZ) & $=\mathbf{1 . 0 4 2}$ & & \\
JIF & $\mathbf{1 . 5 0 0}$ & SJIF (Morocco) $=\mathbf{2 . 0 3 1}$ & & \\
\hline
\end{tabular}

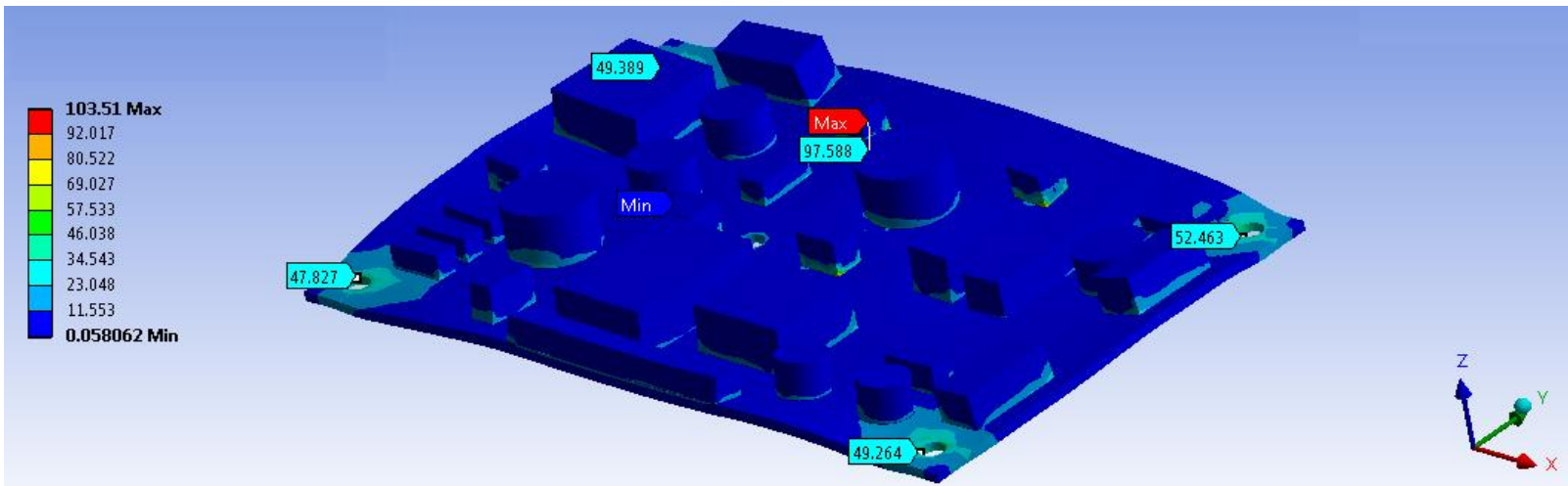

б)

Рисунок 2 - НДС платы при $\mathrm{T}=85^{\circ} \mathrm{C}$ : а) распределение прогибов платы $W$;

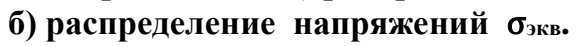

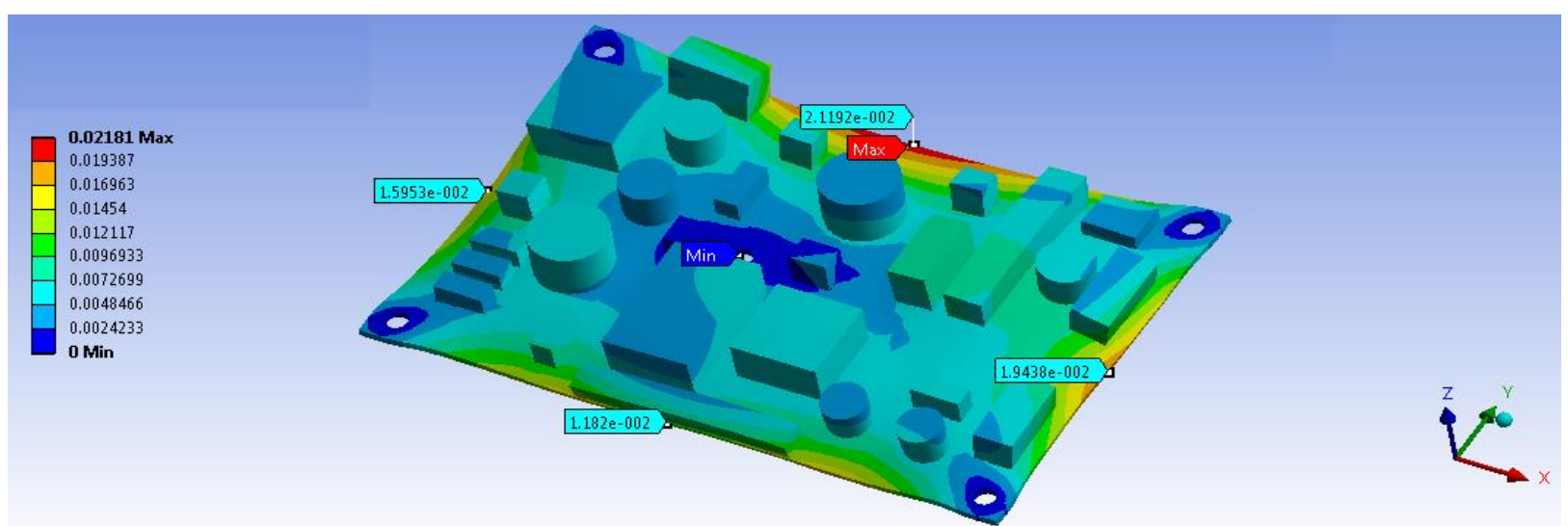

a)

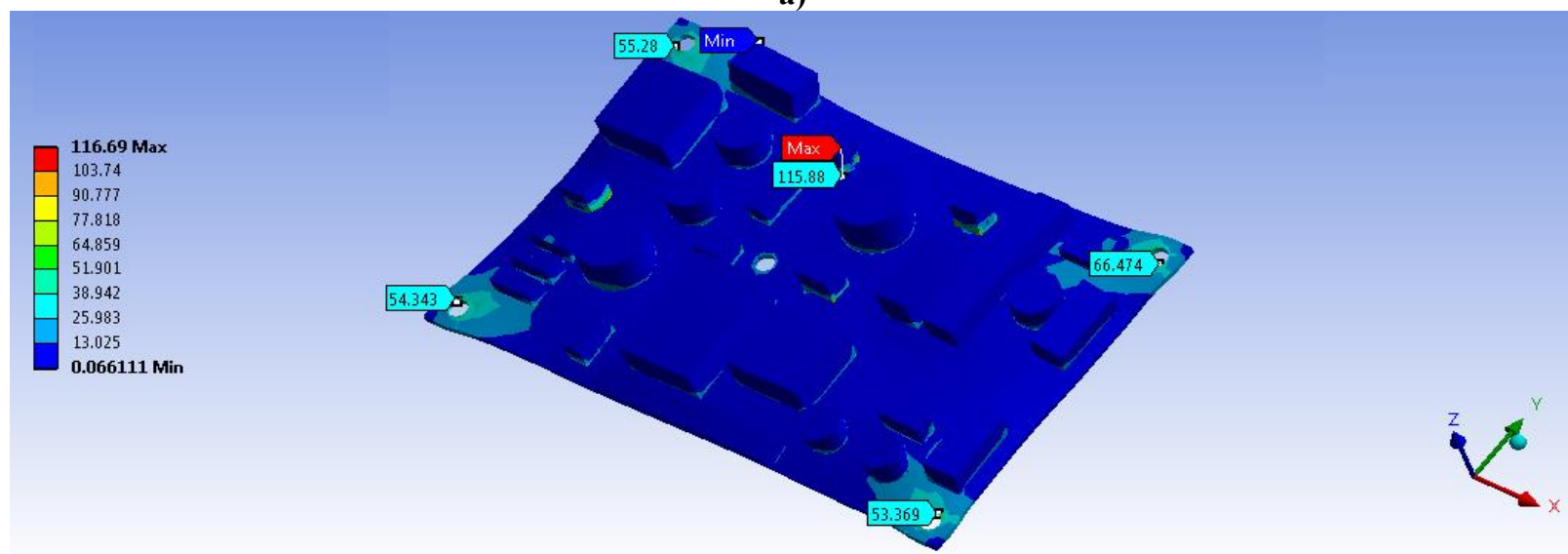

б)

Рисунок 3 - НДС платы при $\mathrm{T}=-$ 50 $^{\circ} \mathrm{C}$ : а) распределение прогибов платы $W$; б) распределение напряжений бэкв. 


\begin{tabular}{l|lr|ll|ll} 
& ISRA (India) & $=\mathbf{1 . 3 4 4}$ & SIS (USA) & $=\mathbf{0 . 9 1 2}$ & ICV (Poland) & $=\mathbf{6 . 6 3 0}$ \\
Impact Factor: & ISI (Dubai, UAE) $=\mathbf{0 . 8 2 9}$ & PUHL (Russia) $=\mathbf{0 . 1 7 9}$ & PIF (India) & $=\mathbf{1 . 9 4 0}$ \\
& GIF (Australia) & $=\mathbf{0 . 5 6 4}$ & ESJI (KZ) & $=\mathbf{1 . 0 4 2}$ & & \\
JIF & $\mathbf{1 . 5 0 0}$ & SJIF (Morocco) $=\mathbf{2 . 0 3 1}$ & & \\
\hline
\end{tabular}

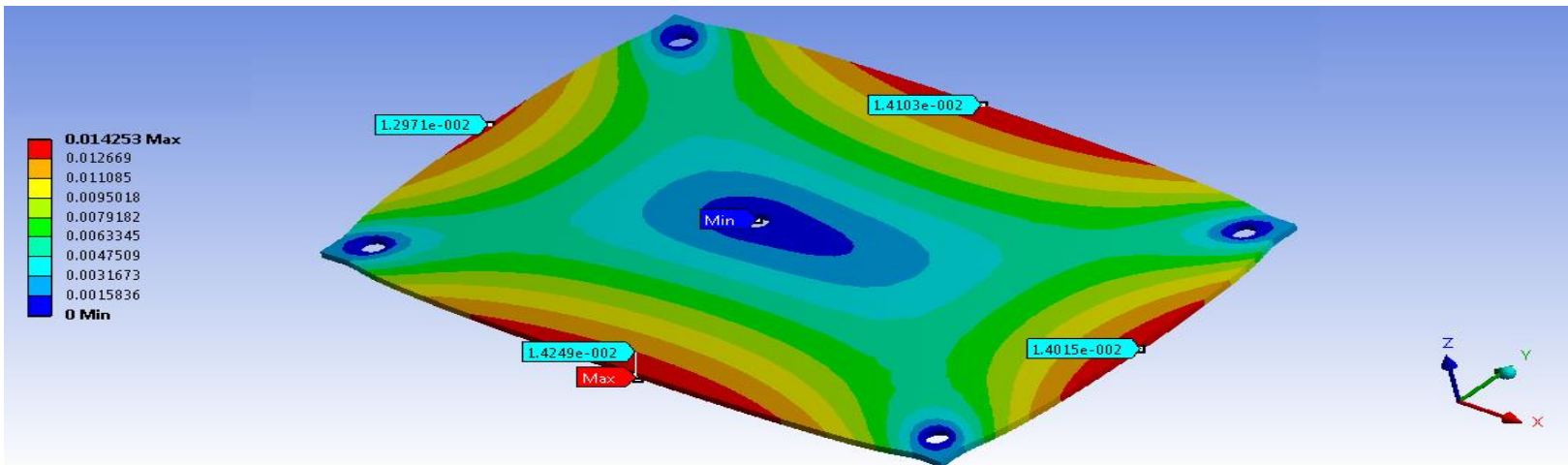

a)

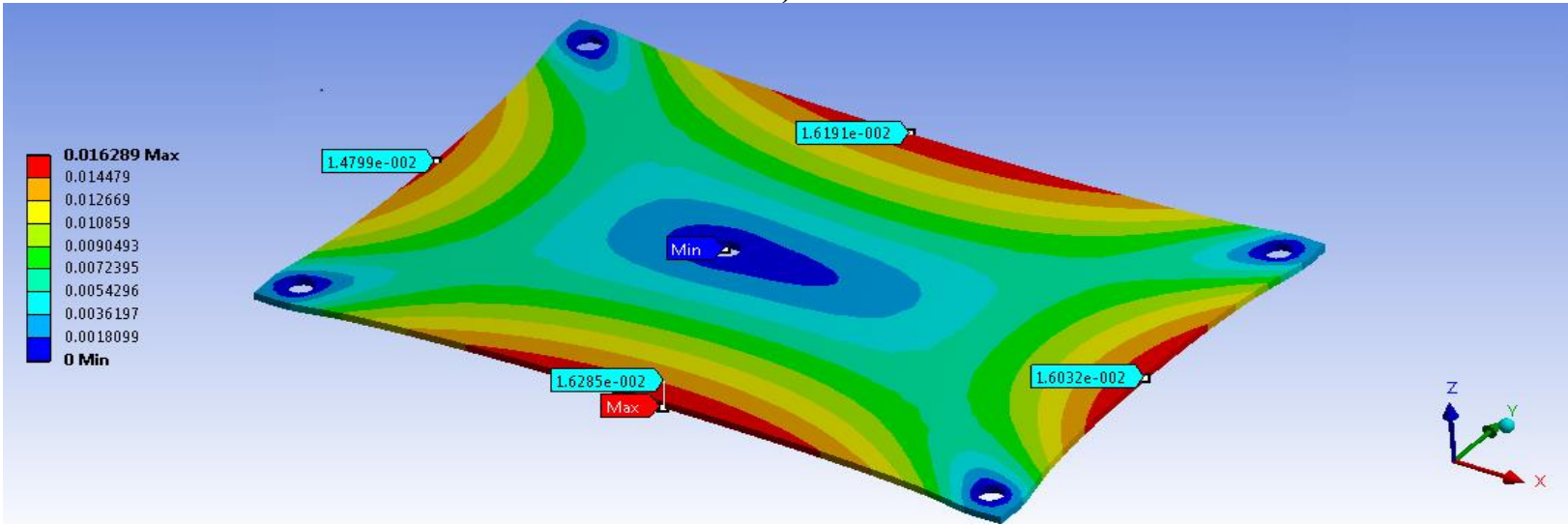

б)

Рисунок 4 - Распределение $W$ по плате: а) при $\mathrm{T}=85^{\circ} \mathrm{C}$; б) при $\mathrm{T}=-50{ }^{\circ} \mathrm{C}$.

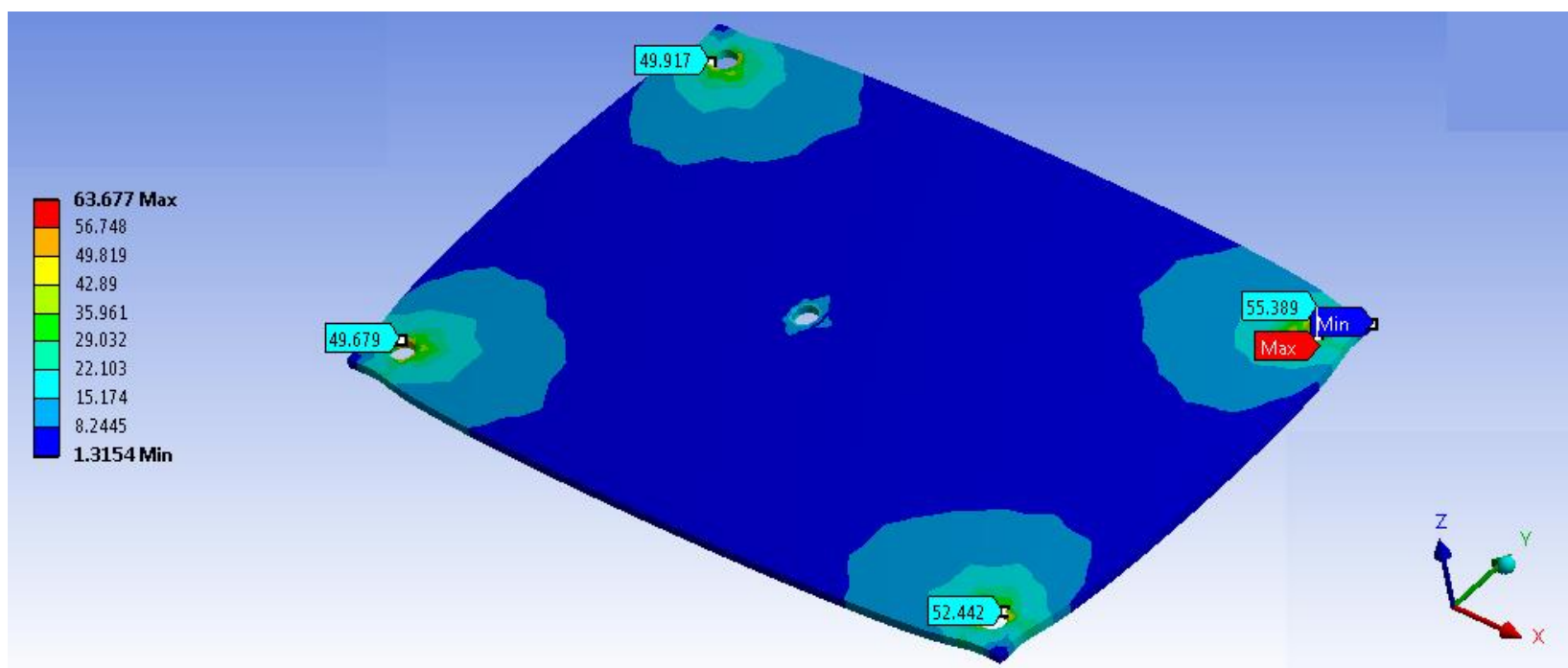

a) 


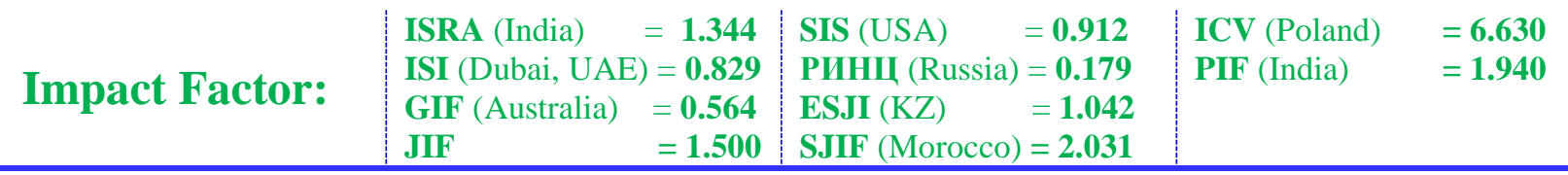

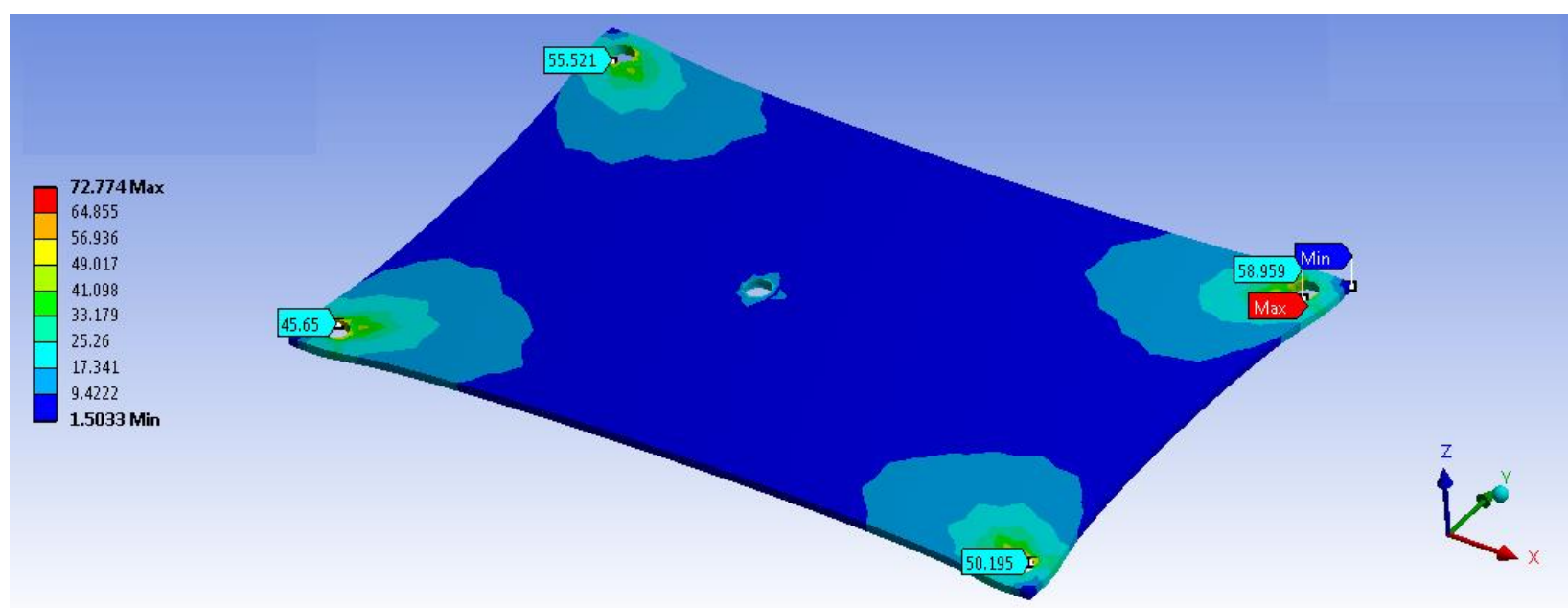

б)

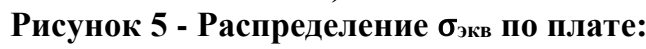
a) при $\mathrm{T}=85^{\circ} \mathrm{C}$; б) при $\mathrm{T}=-50{ }^{\circ} \mathrm{C}$.

Анализ представленных результатов моделирования показывает, что применения второй модели при тепловом воздействии существенно искажает НДС исследуемой ПП. Моделирование НДС ПП при тепловом воздействии для различного расположения ЭРЭ на её поверхности показало, что расположение ЭРЭ существенно влияет на НДС ПП и величины эквивалентных напряжений.

\section{Выводы}

Моделирование НДС для ПП различных типоразмеров при любых способах их закрепления в блоках РЭ показывает, что при тепловых воздействиях происходит их коробление, сопровождающееся ее изгибом.

Установлено, что для адекватного моделирования и анализа НДС плат при тепловых нагрузках рекомендуется применять первую модель, основанную на МКЭ и реализованную в программном комплексе ANSYS, учитывающую реальное расположение и крепление ЭРЭ к плате. Эта модель позволяет определять наиболее нагруженные зоны, в которых возможно образование и развитие дефектов, в том числе и латентных.

Применение

математического моделирования НДС конструктивных элементов блоков РЭ и других приборных устройств на основе рассмотренной модели позволяет на ранних этапах проектирования за счет конструкторско-технологическых решений создавать блоки с повышенной термостабильностью и надежностью в требуемом эксплуатационном режиме внешних воздействий.

С точки зрения дальнейших разработок перспективным следует считать исследование и разработку моделей, позволяющих исследовать НДС элементов РЭ при воздействии тепловых ударов, гармонической и случайной вибрации, линейных и ударных внешних воздействий, а так же их совместного воздействия на проектируемые элементы и блоки РЭ в соответствии с требоваииями, предъявляемыми к надежности и тактико-техническим характеристикам изделий.

\section{References:}

1. Yurkov NK (2014) Technology of production of electronic means: Textbook. / N.K. Yurkov. $\mathrm{SPb}$.: Publisher "Lan", 2014. - 480p.

2. Hadi O.Sh. (2014) Modeling of heterogeneous structures of technical systems to improve safety during their life cycle / O. Sh.Hadi, A.N/ Litvinov // Fundamental problems of system security. Elec, in ESU, .2014. pp.68-75.
3. Litvinov AN (2009) Applied models of heterogeneous structures articles of instrumentation: monograph / A.N.Litvinov, M.A.Litvinov, V.V. Smogunov -Penza Univ PSU 2009-320 p.

4. Litvinov AN (2014) Simulation of stress-strain state of layered structures with RES technology and operational impacts / O. Sh.Hadi, A.N. 
\begin{tabular}{l|lrl|l|ll} 
& ISRA (India) & $=\mathbf{1 . 3 4 4}$ & SIS (USA) & $=\mathbf{0 . 9 1 2}$ & ICV (Poland) & $=\mathbf{6 . 6 3 0}$ \\
Impact Factor: & ISI (Dubai, UAE) $=\mathbf{0 . 8 2 9}$ & PUHIL (Russia) $=\mathbf{0 . 1 7 9}$ & PIF (India) & $=\mathbf{1 . 9 4 0}$ \\
& GIF (Australia) & $\mathbf{0 . 5 6 4}$ & ESJI (KZ) & $=\mathbf{1 . 0 4 2}$ & & \\
\hline JIF & $=\mathbf{1 . 5 0 0}$ & SJIF (Morocco) $=\mathbf{2 . 0 3 1}$ & & \\
\hline
\end{tabular}

Litvinov, N.K.Yurkov // Proceedings of the universities. Volga region. Engineering science, 2014, N4.C.

5. Hadi O.Sh. (2014) Modeling of heterogeneous structures to improve safety during the lifecycle of complex technical systems / O. Sh.Hadi, A.N. Litvinov // Reliability and quality of complex systems, 2014, N 3 (7) .- pp.8-15

6. Bezukhov NN (1968) Fundamentals of the theory of elasticity, plasticity, and creep /N.N.Bezuhov. -Moscow, Graduate School, 1968. -512 p.
7. Kuznetsov OA (1990) The strength of the elements of microelectronic devices / O.A.Kuznetsov, A.I.Pogalov, V.S.Sergeev. Moscow: Radio and communication, 1990.$144 \mathrm{p}$.

8. Hadi A.Sh. (2015) Investigation of the state board electronics with thermal effects / A. Sh.Hadi, A.N. Litvinov, N.K.Yurkov // Proceedings of the universities. Volga region. Tehnicheskie science: Publishing House of the PGU, 2015.-№2. -pp.182-191. 